\title{
Consideraciones históricas y físicas sobre la altura del salto del Tequendama con los datos de Humboldt
}

\author{
Ignacio Mantilla ${ }^{1}$, Fredy Ochoa ${ }^{1, *}$, Roberto Martínez ${ }^{2}$ \\ ${ }^{1}$ Departamento de Matemáticas, Universidad Nacional de Colombia, Bogotá, Colombia \\ ${ }^{2}$ Departamento de Física, Universidad Nacional de Colombia, Bogotá, Colombia
}

\begin{abstract}
Resumen
Se hizo una revisión histórica de los datos reportados por Humboldt para medir la altura del salto del Tequendama mediante el cálculo de la caída de los cuerpos "graves" usando el tiempo de caída e introduciendo diversas correcciones. En condiciones ideales, se verificó que el cálculo coincidía con el valor reportado por Humboldt. Teniendo en cuenta el tiempo que tarda el sonido en regresar a la cima después de que el objeto llega al fondo de la cascada, se obtuvo un error adicional en la medición, lo cual concuerda hasta en una desviación estándar con los datos actuales. Al considerar el efecto de rozamiento del aire sobre el objeto en un régimen turbulento, la estimación se acercó más a las mediciones recientes. (C) 2016. Acad. Colomb. Cienc. Ex. Fis. Nat.
\end{abstract}

Palabras clave: Salto de Tequendama; Caída de los cuerpos.

Some historical and physical aspects of the height of the Tequendama Falls from Humboldt data

\begin{abstract}
We examined the historical description reported by Humboldt to measure the height of the Tequendama Falls using the falling bodies approach. With his data, we estimated the height taking into account different corrections. Under ideal conditions, our calculations were compatible with the value given by Humboldt. We calculated the time sound took from the bottom to the top of the falls and we obtained a value consistent with the current data up to one standard deviation. Our estimations were closer to the most recent data when we took into account friction due to air in a turbulent scenario. (C) 2016. Acad. Colomb. Cienc. Ex. Fis. Nat.
\end{abstract}

Key words: Tequendama falls; Falling bodies.

\section{Introducción}

El salto del Tequendama, cascada natural formada por el río Bogotá en Cundinamarca, se ubica en el puesto 598 a nivel mundial por su altura, calculada en 139 metros. Su majestuosidad, que lo convirtió en sitio sagrado para los Muiscas pobladores de la región en la época precolombina, mereció la mención del naturalista Alexander von Humboldt (Von Humboldt A, 1982), pero se vio afectada por la construcción de la hidroeléctrica de El Charquito en 1900 y del embalse del Muña en 1944, cuyo impacto hizo que el salto perdiera parte de su atractivo natural.

Hoy es todo un reto para los viajeros científicos medir con precisión la altura del salto sin la utilización de técnicas modernas como el láser. Según las mediciones más recientes, se estima que la altura es de 139 m (Otero E., et al., 2010), pero otros cálculos han estimado su altura total en $157 \mathrm{~m}$ (Mendoza A., et al., 1998).

Comparadas con las mediciones más recientes en la cima, las mediciones barométricas realizadas por viajeros de antaño discrepan de forma apreciable, por lo cual medir la altura por diferencia de presión resultaba poco confiable. Las medidas de la profundidad mediante la caída de un cuerpo sólido en las condiciones en que originalmente se hicieron, tampoco habrían resultado muy exactas. No hay un risco que sobresalga lo suficientemente desde la cima para dejar caer limpiamente un objeto, y para eludir los riscos, habría sido necesario arrojar el objeto horizontalmente primero, y hubiera sido difícil establecer si tal lanzamiento no imprimiría una velocidad vertical inicial que modificara la medición final. Se intentaron mediciones mediante triángulos, pero debido a la dificultad del terreno, las mediciones tampoco fueron muy confiables. Una medida generalmente aceptada como confiable fue la obtenida por el Barón Gros y el Coronel Joaquín Acosta utilizando una plomada, lo cual arrojó como resultado una altura de $146 \mathrm{~m}$.

El primer científico que midió la altura del salto del Tequendama fue el sabio Mutis, quien la estableció en 255 varas (214 m); en 1790 el ingeniero Domingo Esquiaqui la

\footnotetext{
*Correspondencia:

Fredy Ochoa, faochoap@unal.edu.co

Recibido: 10 de agosto de 2016

Aceptado: 07 de octubre de 2016
} 
midió en 264,5 varas (222,18 m), en 1801 Humboldt la estimó en 220 varas (184,8 m), y en 1807, el sabio Caldas la calculó en 219 varas (183,96 m) (Díaz S. \& Blanco M., 2010).

Dice el profesor Ignacio Mantilla, rector de la Universidad Nacional de Colombia (Mantilla I., 2016):

“... en efecto, Alexander Von Humboldt fue al Salto del Tequendama el día 27 de agosto de 1801. En su diario escribió: ...el aspecto del Salto es infinitamente bello. Yo creo que no existe ninguna caída de agua de esta altura por la que se precipite tanta agua y en la que se evapore tanta".

Para comprender el texto de Humboldt que se cita a continuación (Von Humboldt A, 1982) es necesario tener en cuenta que un grado centígrado corresponde a 1,25 grados Reaumur, una vara son $0,84 \mathrm{~m}$ y una toesa, $1.949 \mathrm{~m}$.

Dice Humboldt:

"En tiempos del Virrey Espeleta, hombre rígido pero muy activo y útil para el país, le encargó al Brigadier don Domingo Esquiaqui (comandante de la Artillería) de medir el salto para enviar el plano a la Corte. Hizo una medida geométrica desde lo alto, supongo, pero uno de los ángulos del triángulo era inferior a $1^{\circ}$ y los instrumentos con los que operaba tenían errores superiores a los 30'. Hizo sondeos (Sondaleza) y encontró 5 toesas desde arriba hasta el primer escalón, 39 toesas del primer escalón al segundo. El papel periódico de Santa Fé, 1793 , p. 278 , da una medida barométrica del señor Esquiaqui en la cual los datos y el cálculo son tan erróneos que se creería todo suposición sino fuera por el respetable nombre del autor. Barómetro en lo alto del Salto, 23 pulgadas 8 líneas, medida con respecto al Rhin. Abajo: 22 pulgadas 3 líneas. Supongamos que las estaciones superiores e inferiores fueron tomadas una por la otra (aunque el autor agrega que por esas observaciones sabe que la atmósfera abajo es más enrarecida que arriba), la diferencia entre ellas da 289 toesas. Ahora bien, el señor Esquiaqui dice que el río tiene 50 1/2 toesas de desnivel desde la caída (punto donde las aguas caen) hasta el sitio donde tomó la medida; el Salto tendría en consecuencia 239 toesas de altura o sea 3 veces más (Véanse los experimentos sobre la caída de los cuerpos). Pero el autor calcula estas observaciones de otra manera; contando, según dice, 10 toesas por línea encuentra 164 toesas" (Von Humboldt A, 1982, p. 121).

Esto equivale a $319.636 \mathrm{~m}$.

Añade Humboldt en el texto que: “... estuve por la mañana, a las 8 , en zy el termómetro marcó $11.9^{\circ} \mathrm{R}$, descendí al abismo a las $121 / 2$, con cielo sereno y sol radiante, y el mismo termómetro no marcó, en la sombra, sino $12^{\circ} \mathrm{R}$ ", lo cual equivale a medir una temperatura en el salto del Tequendama de $15{ }^{\circ} \mathrm{C}$. Para estar seguro de su medición, Humboldt describe la vegetación y la compara con otros lugares con similar temperatura. Este valor es cercano a los $14{ }^{\circ} \mathrm{C}$ reportados por Mendoza A., et al. (1998), reportes asesorados por Thomas Van der Hammen.

Humboldt señala que,

“... el señor Escallón propone públicamente la tesis de medir desde arriba el Salto. No se necesita ser muy instruido en los elementos de la geometría para entrever esta posibilidad. Podemos imaginar arriba, en z, una base horizontal o perpendicular, medir los ángulos de depresión... Arrojar al abismo una vara de longitud conocida y medirla desde arriba... Todo esto sería exacto si tuviéramos instrumentos que dieran 1" de exactitud, bases con precisión de 2 líneas. Pero esta ejecución en un terreno, en z (en lo alto de la cascada) que no permite sino una base de 20 pies de longitud, es como medir la distancia de la Tierra a la luna tomando los ángulos desde los dos extremos de una casa. Llevé al Tequendama un grafómetro y un sextante pero no intenté tomar medida alguna. Imaginémonos situados sobre el borde de un muro de 90 a 100 toesas de altura, distante 7 a 8 toesas de un muro paralelo, tratando de medir la profundidad del intersticio, teniendo en cuenta que, desde arriba, el muro no permite una base de más de 4 toesas de largo y que no se aprecian desde allí sino 30 a 40 toesas de longitud en el intersticio. Es lo mismo que colocarse al borde de un pozo dentro del cual se ha introducido una lucecita e imaginar que se puede medir trigonométricamente la profundidad de esta luz. La medida desde abajo es aún menos factible. No se puede uno acercar al punto y, ni se puede ver, siquiera de lejos, el pie de la cascada. Confiaba yo mucho en esta última posibilidad. Viendo y desde lejos subiría y bajaría por la pendiente de la montaña de la Povasa hasta que el nivel me indicara que estaba a la misma altura de y. Así, la estación barométrica del punto hallado a la misma altura de $\mathrm{y}$, comparada con la altura barométrica de z, daría la altura de la cascada, zy, corrigiéndola por la curvatura de la Tierra. Era asociar un método barométrico con un nivel buscado geodésicamente. Los habitantes niegan la posibilidad de tal perspectiva. Es cierto que el Sagasugá es tan sinuoso que no se puede ver el pie del Salto, punto y. Sin embargo, creo que caminando largo rato por la pendiente del peñasco, exponiéndose al peligro y tumbando los árboles, se podría encontrar tal vez la perspectiva buscada." (Von Humboldt A, 1982).

Humboldt explica la dificultad de usar este método por las condiciones escabrosas del terrero, lo cual dificultaría enormemente construir los triángulos y hacer mediciones, y por ello, lo descartó.

Por último, Humboldt presenta el método que considera más confiable para medir la altura del salto de Tequendama:

“... caída de los cuerpos. He aquí el método en que más confió. Hice lanzar más de 15 veces piedras en el precipicio, diciendo tac cuando la piedra caía perpendicularmente sobre el abismo y tac cuando llegaba al fondo, a la superficie del agua. Tenía el cronómetro a la mano y numerosos espectadores, suficientemente instruidos, juzgaban si el experimento estaba bien o mal hecho. Se distingue fácilmente $1 / 5$ de segundo. En todas las caídas no hubo sino 0,4 segundos de incertidumbre, prueba de que los experimentos estaban bien hechos. El tiempo promedio de la caída fue de 6,4 segundos lo que da, por el cuadrado de tiempo, 108 toesas de profundidad, pero creo que la piedra descendía demasiado lentamente. No se 
la puede hacer caer perpendicularmente, sin impulso. La forma del peñasco lo impide. Para que caiga al fondo del abismo hay que lanzarla con fuerza. En consecuencia, la piedra cae según una parábola por la fuerza horizontal que se le ha dado. Esta fuerza disminuye la de gravedad. Nos encontramos frente a un máximo. Hay razones, tal vez, para que la piedra descienda lentamente pero no hay ninguna (la tendría en cuenta) para que caiga demasiado rápido. La cascada no puede tener más de 108 toesas pero su altura es probablemente menor. La fuerza de proyección horizontal, ¿sería de 1/6 del tiempo? ¿Cómo medirla? Como la piedra se arroja bastante lejos sin gran impulso (se ha tratado de darle el impulso que parece suficiente para que llegue al punto deseado), hay que creer que la fuerza (el impulso horizontal) está casi anulada cuando la piedra empieza a caer. Yo creo que se pueden contar 6" de caída lo cual da zy $=93$ toesas. El señor Cabrer, Oficial Ingeniero muy instruido, hizo antes que yo los mismos experimentos y dedujo (por lo que me dijo) 115 toesas sin descontar nada por el impulso horizontal." (El subrayado es nuestro) (Von Humboldt A, 1982).

Humboldt reporta dos valores para la altura medidos mediante la caída de los cuerpos graves: el valor de 108 toesas que él midió, el cual equivale a $210,492 \mathrm{~m}$, y el valor de 115 toesas que reportó su ingeniero con algunas correcciones explicadas por él, el cual equivale a 224,135 m.

Humboldt también midió con un barómetro la altura del salto siguiendo el siguiente procedimiento (Boussingault J.B \& Koppel de León A., s.f): de acuerdo con las observaciones barométricas, el fondo del canal está 183 metros más bajo que el río Bogotá en el Puente del Común, es decir a una altura de $2.605 \mathrm{~m}$, y la altura del salto del Tequendama es de $2.422 \mathrm{~m}$, por lo tanto, la diferencia es de 183 metros, los cuales equivalen a 94 toesas.

\section{Estimación de la altura del salto según los datos de Humboldt}

En este trabajo se propuso estimar la altura del salto del Tequendama considerando las mediciones reportadas por Humboldt, quien midió un tiempo de caída (t) de 6,4 segundos y un error $(\boldsymbol{\Delta t})$ de 0,4 segundos. Si se asume que la piedra se dejó caer verticalmente, y se desprecia el efecto del rozamiento del aire, la expresión de la altura en función del tiempo es:

$$
h=\frac{1}{2} g t^{2}
$$

Usando el método de elementos finitos para el error en la altura, se obtiene la expresión:

$$
\Delta h=g t \Delta t
$$

Tomando el valor de la aceleración de la gravedad, $g=$ $9,8 \mathrm{~m} / \mathrm{s}^{2}$, se obtiene:

$$
h=200,7 \mathrm{~m} \text { y } \Delta h=25 \mathrm{~m}
$$

lo cual equivale a una altura de 103 toesas con un error de 13 toesas. Esta estimación está de acuerdo con el valor estimado por Humboldt de 108 toesas y el de su ingeniero, de 115 toesas. La velocidad final, antes de tocar el fondo, es de $v=g t=62 \mathrm{~m} / \mathrm{s}$. Por esta razón, un pequeño error en la medición del tiempo se traduce en una enorme distancia recorrida en el tramo final.

\section{Estimación corregida de la altura del salto con los datos de Humboldt}

Usando los mismos datos que presenta Humboldt, se quiso estimar con mayor precisión la altura del salto del Tequendama. Para ello era necesario tener en cuenta otros errores en la medición del tiempo; además del reportado por Humboldt, el cual se denotó como $\Delta t=0,4$ segundos, se consideró el retraso debido a la velocidad finita del sonido en el aire entre la llegada de la piedra al fondo y el registro del 'tac' en la cima, el cual se denominó como $\Delta t$ '. Si $h$ representa la altura del salto, y $c$ la velocidad del sonido en el aire, entonces:

$$
\Delta t^{\prime}=\frac{h}{c}
$$

La velocidad del sonido a $0{ }^{\circ} \mathrm{C}$, es $c=331 \mathrm{~m} / \mathrm{s}$. Pero es posible mejorar la precisión si se considera la corrección de la velocidad del sonido debido a la temperatura, correspondiente a unos $0,6 \mathrm{~m} / \mathrm{s}$ por cada grado centígrado de aumento en la temperatura. Teniendo en cuenta la temperatura de $15{ }^{\circ} \mathrm{C}$ reportada por Humboldt, se obtiene una velocidad del sonido de $c=340 \mathrm{~m} / \mathrm{s}$. Al incluir el tiempo de retraso del 'tac', el tiempo real de caída es:

$$
T=t-\Delta t^{\prime},
$$

siendo $t$ el tiempo medido por Humboldt. En este escenario, la expresión de la altura en función del tiempo se convierte en:

$$
\begin{aligned}
h & =\frac{1}{2} g T^{2}=\frac{1}{2} g\left(t-\Delta t^{\prime}\right)^{2}=\frac{1}{2} g\left(t-\frac{h}{\mathrm{c}}\right)^{2} \\
& \approx=\frac{1}{2} g t^{2}-\frac{g t}{\mathrm{c}} h
\end{aligned}
$$

donde se han despreciado términos de segundo orden en $\Delta t^{\prime}$ $=\frac{h}{\mathrm{c}}$. Así, al despejar la altura, se obtiene:

$$
h=\frac{g^{2} / 2}{1+g t c}
$$

El factor $1 /(1+g t / c)$ se interpreta, entonces, como la corrección debida a la demora del sonido en regresar a la cima para completar la medida del tiempo. El valor estimado para este factor es de 0,84 , lo cual equivale a una corrección del valor reportado por Humboldt del orden de $16 \%$. Entonces:

$$
h=168,6 \mathrm{~m} ; \Delta h=25 \mathrm{~m} \text {, }
$$

lo cual expresado en toesas es:

$$
h=86,5 \text { toesas; } \Delta h=13 \text { toesas. }
$$

Si en la ecuación (4) se aplicara un tratamiento exacto debido al error cometido en el tiempo de regreso de la señal sin despreciar términos de orden superior, el cálculo de la altura vendría dado por la ecuación cuadrática:

$$
\frac{g}{2 \mathrm{c}^{2}} h^{2}-\left(1+\frac{g t}{\mathrm{c}}\right) h+\frac{1}{2} g t^{2}=0
$$

la cual exhibe las dos soluciones algebraicas conocidas, una con términos que suman y la otra con los que restan. Solamente la solución que resta reproduce el límite $h=\frac{1}{2} g t^{2}$ cuando $\Delta t^{\prime}=0$, para así obtener:

$$
h=\frac{c^{2}}{g}\left[1+\frac{g t}{c}-\sqrt{\left(1+\frac{g t}{c}\right)^{2}-\left(\frac{g t}{c}\right)^{2}}\right]
$$


Para calcular el error en la altura se tomó el segundo orden de la expansión de Taylor de la función $h(t+\Delta t)$ alrededor de $t$ :

$$
\begin{aligned}
& \Delta h=h(t+\Delta t)-\mathrm{h}(t) \\
& =\frac{\partial h}{\partial \mathrm{t}} \Delta \mathrm{t}+\frac{1}{2 !} \frac{\partial^{2} h}{\partial t} \Delta t^{2}
\end{aligned}
$$

Por lo tanto, mediante la expresión (7), se obtiene:

$$
\Delta h=c\left(1-\frac{1}{\sqrt{1+\frac{2 g t}{c}}}\right) \Delta t+\frac{1}{2 !} \frac{g}{\left(1+\frac{2 g t}{c}\right)^{3 / 2}} \Delta t^{2}
$$

Con estas nuevas expresiones, y usando los datos reportados por Humboldt, se llegó al siguiente resultado:

$$
h=170,5 \mathrm{~m} ; \Delta h=20,3 \mathrm{~m} \text {. }
$$

\section{Efecto del aire en la caída de los cuerpos graves}

Un efecto que no se ha tenido en cuenta en la caída de los objetos que lanzaba Humboldt es la fricción con el aire. Debido el gran caudal del salto y la velocidad que el agua adquiere al caer, cerca de $190 \mathrm{~km} / \mathrm{h}$ al final del tramo, se forma un rocío de gotas de agua cuando esta golpea las paredes de piedra de la cascada. Este rocío se combina con el aire formando una niebla de aire húmedo, lo cual produce fricción en la caída del objeto y podría introducir otro factor de corrección en la medida de la altura.

En el escenario más simple de un flujo laminar alrededor de un objeto esférico, la fuerza de fricción está descrita por la ley de Stokes:

con:

$$
F=-b \frac{d h}{d t}
$$

$$
b=6 \pi R \mu \text {, }
$$

donde $R$ es el radio del objeto y $\mu$ la viscosidad del aire húmedo. Al sumar el resultado con la fuerza de la gravedad e integrar la ecuación de movimiento, suponiendo que la velocidad inicial sea cero, se obtiene para la altura:

$$
h=\left(\frac{m g}{6 \pi R \mu} t\right)+\left(\frac{m}{6 \pi R \mu}\right)^{2} g\left(e^{-\frac{6 \pi R \mu}{m} t}-1\right)
$$

Considerando la expansión de potencias en la función exponencial, se obtiene:

$$
\begin{aligned}
h & =\frac{m g}{6 \pi R \mu} t+\left(\frac{m}{6 \pi R \mu}\right)^{2} g\left(-\frac{6 \pi R \mu}{m} t+\frac{1}{2 !}\left(\frac{6 \pi R \mu}{m} t\right)^{2}+\mathcal{O}\left[\mu^{3}\right]\right) \\
& =\frac{1}{2} g t^{2}+\mathcal{O}[\mu]
\end{aligned}
$$

por lo que en el límite $\mu \rightarrow 0$, se reproduce la expresión para la caída libre de la ecuación (1). Para usar la ecuación (10), se requiere el valor de la masa de las rocas lanzadas por Humboldt, sobre el cual carecemos de información. Sin embargo, se puede estimar si expresamos la masa en términos de la densidad de masa $\rho$, con lo cual para la altura se obtiene:

$$
h=\frac{2 \rho R^{2} g}{9 \mu} t+\left(\frac{2 \rho R^{2}}{9 \mu}\right)^{2} g\left(e^{-\frac{9 \mu}{2 \rho R^{2}} t}-1\right)
$$

Se asumió una densidad de $\rho=2.200 \mathrm{~kg} / \mathrm{m}^{3}$, correspondiente a las rocas de tipo arenisca encontradas en el salto. Para el radio $R$, se contempló utilizar desde una piedra que cupiera en una mano hasta una roca que se pudiera lanzar horizontalmente con la mano. Se estimó un rango $R=0,04$ - 0,1 m. En cuanto a la viscosidad, se empleó la del aire húmedo, la cual se estima en el orden de $\mu=1,8 \times 10^{-5} \mathrm{~kg} /$ $\mathrm{m} \cdot \mathrm{s}$, de manera que el factor que acompañara el tiempo en la exponencial de (11) fuera del siguiente orden:

$$
\frac{9 \mu}{2 \rho R^{2}}=2.3 \times 10^{-5}-3.7 \times 10^{-6} \mathrm{~s}^{-1},
$$

el cual es varios órdenes de magnitud menor al tiempo de caída, por lo que era razonable considerar una expansión en series de potencias de la exponencial, para así obtener:

$$
h \approx \frac{g t^{2}}{2}-\frac{3 g \mu t^{3}}{4 \rho R^{2}}
$$

donde la contribución debido al rozamiento del aire era apenas de $0,16 \mathrm{~cm}$ a $1 \mathrm{~cm}$, lo cual es claramente despreciable. Una aproximación más realista era considerar que el flujo alrededor de la piedra al caer era turbulento, en cuyo caso la fuerza de fricción aumentaría proporcionalmente al cuadrado de la velocidad, así (Lindemuth J., 1971):

$$
F=-\frac{1}{5} \rho_{\text {aire }} R^{2}(d h / d t)^{2} \text {. }
$$

Una solución aproximada de la ecuación de movimiento dio lo siguiente:

$$
\begin{aligned}
h & \approx \frac{1}{2} g t^{2}-\frac{\pi}{60 m} \rho_{\text {aire }} g^{2} R^{2} t^{4} \\
& \simeq \frac{1}{2} g t^{2}-\frac{\rho_{\text {aire }}}{80 R \rho} g^{2} t^{4}
\end{aligned}
$$

En este caso, la corrección de la altura debido al efecto turbulento sería del orden de $11 \mathrm{~m}$ con una roca de $0,1 \mathrm{~m}$ de radio y tomando $\rho_{\text {aire }}=1,2 \mathrm{~kg} / \mathrm{m}^{2}$.

Teniendo en cuenta el efecto sonoro para la medición del tiempo y los efectos viscosos, la predicción que obtendríamos sería:

$$
h=162,4 \mathrm{~m} ; \Delta h=19,8 \mathrm{~m} .
$$

\section{Medición de Humboldt con correcciones aerodinámicas}

Las correcciones aerodinámicas introdujeron una corrección de $11 \mathrm{~m}$; si se supone que el tiempo medido es solo el de la caída del cuerpo, entonces la medición de Humboldt con el tiempo reportado debería ser de:

$$
h=189,7 \mathrm{~m} ; \Delta h=24 \mathrm{~m} \text {. }
$$

\section{Conclusiones}

Aún hoy en día sigue siendo un reto medir la altura del salto del Tequendama. Con las técnicas más sofisticadas de interferometría láser es necesario bajar al fondo del salto para colocar un espejo que refleje la luz, lo cual es posible solo para expertos que puedan bajar con cuerdas por riscos de 100 metros de profundidad. En la visita que hicimos al salto pudimos entender mejor los diferentes métodos usados y las dificultades para medir su altura. Según la descripción de Humboldt, con el método de la caída de los graves no quedó claro si la medición del tiempo se hizo con señas para indicar cuándo salía y cuándo caía la piedra al fondo, lo cual puede introducir una incertidumbre grande. La otra forma sería medir el momento de salida de la piedra y de la señal sonora cuando golpea en el fondo. Sin embargo, 
debido al alto caudal del salto, la señal sonora podía quedar amortiguada por el ruido del agua, lo cual haría muy difícil la medición.

Teniendo en cuenta las correcciones y el tratamiento de los errores en las mediciones del tiempo, fue posible obtener unas mediciones de la altura del salto del Tequendama más cercanas a los reportadas recientemente, incluso con los datos obtenidos por Humboldt, lo cual indica que sus mediciones, a pesar de las dificultades, son aceptables.

Si consideramos las mediciones más recientes de la altura del salto, de $139 \mathrm{~m}$ o $157 \mathrm{~m}$, se concluye que el valor obtenido utilizando los datos de Humboldt con una corrección de segundo orden debido al retraso del sonido, es decir, $168,6 \pm 25 \mathrm{~m}$, concuerda con los $157 \mathrm{~m}$ y media desviación estándar y con los $139 \mathrm{~m}$ y una desviación estándar. Sin embargo, del análisis podemos concluir que para la época no era claro el tiempo de retardo debido a la demora de la señal en regresar, a pesar de que ya se conocía la velocidad del sonido. Tampoco es claro en la descripción de Humboldt si se tuvo en cuenta este retardo. Dichas correcciones tienen una incidencia importante en la predicción de la altura usando la caída de los graves. Con base en las soluciones exactas, la altura predicha se modificó y aumentó dos metros, a 170,5 $\mathrm{m}$, y el error en la altura se redujo en un $12 \%$ a 19,8 m.

Teniendo en cuenta el efecto del aire en un escenario turbulento, la altura estimada se pudo reducir hasta 162,4 m. La apreciación de Humboldt de que "la piedra parecía descender demasiado lentamente" podría tener un fundamento físico si se tiene en cuenta el retraso debido al tiempo que tarda el sonido en volver a la cima y el rozamiento del aire que reduce la aceleración de los objetos.

Por último, hay que considerar que en el presente análisis se asumió que la posición del borde de la cascada hoy es la que tenía en la epoca de las mediciones de Humboldt. Sin embargo, desde el punto de vista geológico, la corriente de agua de la cascada puede haber producido una erosión apreciable, disminuyendo la altura del borde en un orden de hasta un metro, y en ese caso, el error de las estimaciones de este estudio pudo incrementarse en ese mismo orden.

Durante la visita al salto del Tequendama pudimos conversar con bomberos que estaban haciendo trabajos de entrenamiento y nos dijeron que ellos han medido con cuerdas la altura del salto y han obtenido 147 m. Así, los datos del Barón Gros y Joaquín Acosta, quienes establecieron la altura en $146 \mathrm{~m}$ utilizando una plomada, parece ser el valor más confiable.

\section{Agradecimientos}

Agradecemos el apoyo del programa de Colciencias: El Patrimonio Autónomo Fondo Nacional de Financiamiento para la Ciencias, la Tecnología y la Innovación Fransisco José de Caldas, contrato No. FP44842-050-2015.

\section{Conflicto de intereses}

Los autores manifiestan que no tienen conflicto de intereses.

\section{Referencias}

Von Humboldt A. 1982. Academia Colombiana de Ciencias Exactas, Físicas y Naturales. Alexander von Humboldt en Colombia. Extractos de sus diarios. Academia Colombiana de Ciencias Exactas, Físicas y Naturales. Bogotá - Colombia. p. 1-247. Disponible en: http://www.comunidadandina.org/bda/docs/ CO-CA-0004.pdf

Boussingault J.B \& Koppel de León A. (s.f). Memorias. Cap. XII, el salto de Tequendama - Historia de Manuelita Sáenz. Biblioteca Virtual del Banco de la República. Disponible en: http://www.banrepcultural.org/blaavirtual/historia/memov1/ memov13a.htm. [2016, 5 de Junio].

Díaz S. \& Blanco M. 2010. Rescatan memoria del salto de Tequendama. Periódico El Tiempo. Disponible en: http:// www.eltiempo.com/archivo/documento/MAM-4124046. [2010, 2 de septiembre].

Lindemuth J. 1971. The effect of fair resistance on falling balls. Am. J. Phys. 39: 757-759. Disponible en: http://www.sc. ehu.es/sbweb/fisica_/dinamica/fluidos/stokes1/stokes1_3. html. [2016, 12 de Junio].

Mantilla, I. 2016. El Salto de Tequendama, un salto a la ciencia. Periódico El Espectador. Disponible en: http://www. elespectador.com/opinion/el-salto-de-tequendama-un-saltociencia. [2016, 3 de junio].

Mendoza A., García D., Escorcia W. 1998. Cuenca alta del río Bogotá. Descripción y diagnóstico. Sociedad Geográfica de Colombia, Academia de Ciencias Geográficas. Bogotá - Colombia. p. 7.

Otero E., Mosquera L., Silva G., Guzmán J. 2010. Saltos, Cascadas y Raudales de Colombia. Capítulo 4. Libros de la Colección Ecológica del Banco de Occidente. Cali, Colombia. p. 12. Disponible en: http://www.imeditores. com/banocc/saltos/presentacion.htm [2016, 12 de Junio]. 